\title{
Laparoscopic partial nephrectomy of thyroid cancer metastasis: case report and review of the literature
}

This article was published in the following Dove Press journal:

OncoTargets and Therapy

9 April 2013

Number of times this article has been viewed

\author{
Giovanni Cochetti' \\ Efisio Puxeddu \\ Michele Del Zingaro 3 \\ Francesco D'Amico' \\ Emanuele Cottini' \\ Francesco Barillaro' \\ Ettore Mearini' \\ 'Department of General Surgery \\ and Surgical Specialties, Urological \\ Andrological Surgery and Minimally \\ Invasive Techniques, University of \\ Perugia, Terni, Italy; ${ }^{2}$ Department \\ of Internal Medicine, ${ }^{3}$ Department \\ of Surgical Specialties and Public \\ Health, Urological Clinic, University \\ of Perugia, Perugia, Italy
}

Background: Follicular cell thyroid carcinoma is a quite aggressive form of thyroid cancer. About $10 \%$ of follicular thyroid carcinoma shows multiple metastases: lung and bone are the most common sites of metastasis. Renal involvement from thyroid primary cancer is very rare with incidence of $4.5 \%-5.9 \%$.

Purpose: We report the first laparoscopic conservative treatment of renal metastasis from thyroid cancer. This is a new and useful approach in order to delay malignant disease progression and to reduce the surgical discomfort of the patient.

Patients and methods: We present the case of a 67-year-old woman, undergoing total thyroidectomy for follicular thyroid cancer with bone and lung metastasis. During adjuvant radiometabolic treatment, renal metastasis was diagnosed. Renal metastasis showed high metabolic activity, reducing the effectiveness of radioiodine therapy for secondary lesions. For this reason, we performed a laparoscopic simple enucleation of the single renal metastasis using extraperitoneal access and a clampless procedure.

Results: The excision of the renal lesion improved the effectiveness of adjuvant radioiodine therapy: two months after surgery, the patient underwent adjuvant radiometabolic treatment with iodine-131 (150 mCi) and the following whole body scan showed only a small uptaking area at the level of the vertebral metastasis. The lung micrometastases were not detectable. At 36 months follow-up, malignant disease was clinically stable and well controlled.

Conclusion: Minimally invasive renal surgery with preservation of renal function and rapid recovery contributed to the success of radioiodine therapy and delayed the progression of the disease.

Keywords: follicular thyroid carcinoma, renal metastasis, simple enucleation, laparoscopy, clampless, radiometabolic treatment

\section{Introduction}

Despite the high frequency of nodular thyroid disease, malignancies are not common $(3 \%-5 \%)$. Thyroid carcinoma represents $1.6 \%$ of all male cancers and $5 \%$ of female ones. ${ }^{1}$ The incidence is 41 cases per 10,000,000 men and 100-120 cases per 10,000,000 women between the fifth and eighth decade. ${ }^{2}$ The American Cancer Society has estimated 56,460 expected new cases of thyroid carcinoma in the US in 2012 and 1780 expected thyroid cancer related deaths. ${ }^{1}$ In 2009 in the US, about 1600 deaths occurred in about 300,000 patients affected by this tumor. ${ }^{2}$

Histopathologically, most thyroid cancers are well-differentiated. Generally the prognosis is favorable and the survival rate is over $90 \%$ at 10 years after diagnosis. Among the well-differentiated forms, follicular carcinoma is the most aggressive
Correspondence: Giovanni Cochetti Department of Surgical Specialties and Public Health; Division of Urological Andrological Surgery and Minimally Invasive Techniques, Terni, University of Perugia, I 5 Via Billè 63900 Fermo, Italy Tel +39074420 5822

Fax +39074420 5822

Email giovannicochetti@libero.it 
because it has a propensity for vascular invasion and hematogenous spread. The most common sites of metastasis are lung and bone, less frequently brain, liver, skin, kidney, and adrenal gland. ${ }^{3-5}$ However, overall survival at 5, 10, and 20 years for patients with follicular thyroid cancer is $85 \%$, $80 \%$, and $76 \%$, respectively. 5,6

We present the case of a 67-year-old female, undergoing total thyroidectomy for follicular thyroid cancer metastasizing bone. During adjuvant radiotherapy, renal metastasis was diagnosed. We performed laparoscopic extraperitoneal simple enucleation of the renal lesion in order to allow more rapid recovery of general health and to ensure more effective and earlier adjuvant radiometabolic treatment.

\section{Material and methods}

Due to chest pain, a 67-year-old woman underwent a chestabdomen computed tomography (CT) scan, that showed osteolysis of the soma of D3 with complete destruction of the posterior wall, infiltration of the spinal canal, and some low-density nodular opacities in both lungs. The biopsy of the bone injury showed wide infiltration by tumoral cells with the pattern of follicular thyroid cancer. After 16 Gy local radiotherapy, magnetic resonance imaging confirmed the findings of the CT scan and also detected similar injury at the level of D4. Therefore, the patient underwent decompressive laminectomy of the D3-D4 tract and stabilization of the D1-D6 tract. Furthermore, total thyroidectomy was performed due to the presence of a $1.1 \mathrm{~cm}$ left lobe thyroid nodule. Histological examination revealed follicular thyroid carcinoma (pT1bN0M1, stage IVC, according to the 2009 tumor, node, metastasis [TNM] classification). ${ }^{7}$ Immunohistochemical analysis confirmed the histological findings, showing positive results for galectin-3, HBME1, and CK19.

After thyroidectomy, serum thyroglobulin was $1339 \mathrm{ng} / \mathrm{mL}$, thyroid-stimulating hormone (TSH) under the lack of L-thyroxine was $0.3 \mathrm{mU} / \mathrm{L}$ (range $0.4-4.0 \mathrm{mU} / \mathrm{L}$ ), and antithyroglobulin antibody was negative.

After 3 months, the patient underwent radiometabolic treatment with $1852 \mathrm{MBq}(50.05 \mathrm{mCi})$ of iodine-131 $\left({ }^{131} \mathrm{I}\right)$. The following single-photon emission CT and ${ }^{131} \mathrm{I}$ whole body scan (WBS) showed two large foci of increased uptake at the D3 vertebral soma and the lower pole of the left kidney, both reported to be metastases (Figures 1 and 2). An abdominal CT scan showed a hypodense area of $2 \mathrm{~cm} \times 2 \mathrm{~cm}$ at the lower pole of the left kidney with obvious contrast enhancement. In order to obtain a debulking of the surgically removable tumor mass, we performed a laparoscopic

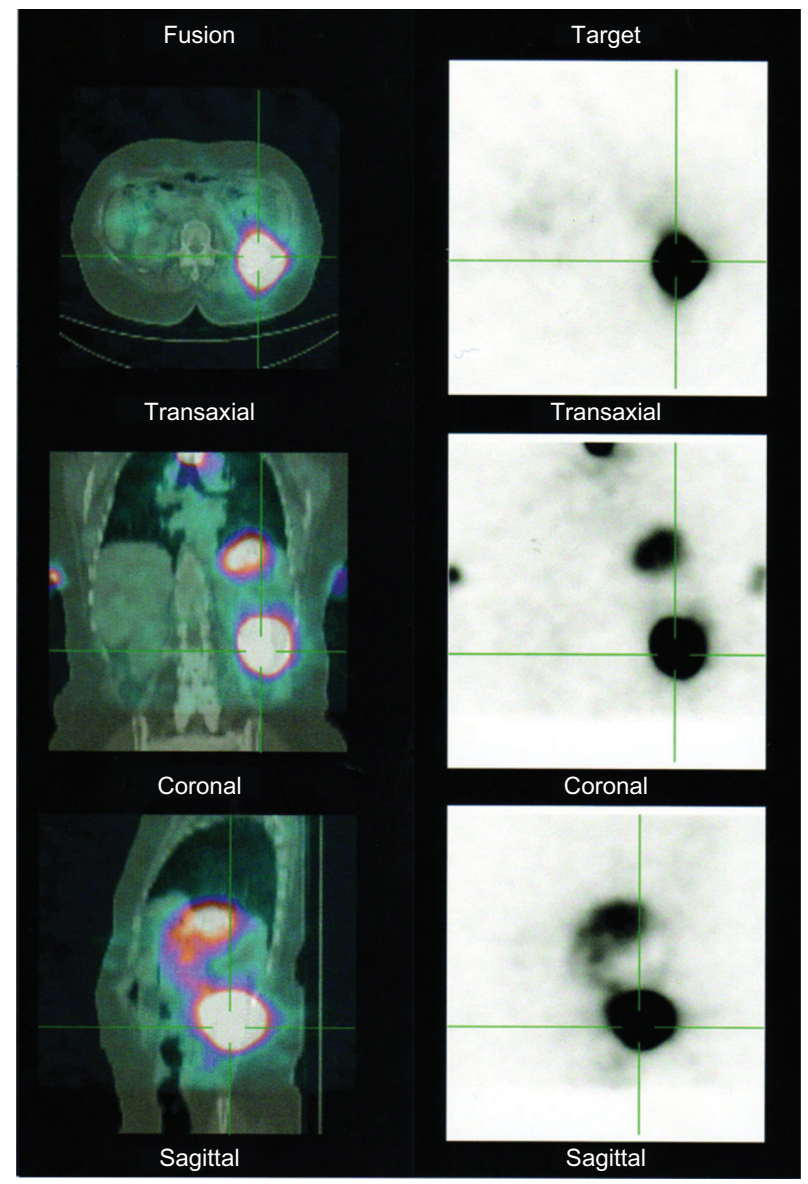

Figure I SPECT.

Notes: The exam was performed during the first radiometabolic treatment and before simple enucleation; it showed high uptake at the lower pole of the left kidney against a common and diffuse background of high gastric and intestinal radioiodine activity.

Abbreviation: SPECT, single-photon emission computed tomography.

simple enucleation of the renal tumor (Figure 3), using extraperitoneal access and a clampless procedure: the renal artery was only isolated and dissected, avoiding ischemia of the healthy tissue. Hemostasis was achieved using a combination of radiolucent clips (Weck Hem-o-lock clips; Teleflex Incorporated, Limerick, PA, USA), intracorporeal suturing, and hemostatic material. Surgery was considered necessary in order to reduce the tumor burden to be treated by radioactive iodine and to increase the chance of its efficacy of vertebral and lung metastases treatment.

\section{Results}

Estimated blood loss during surgery was $100 \mathrm{~mL}$ and the operative time was 180 minutes. The length of stay was 6 days. Serum creatinine was constantly $0.7 \mathrm{mg} / \mathrm{dL}$ and the estimated glomerular filtration rate was $88.61 \mathrm{~mL} /$ minute. There were no postoperative complications. Histological examination confirmed the presence of follicular thyroid 


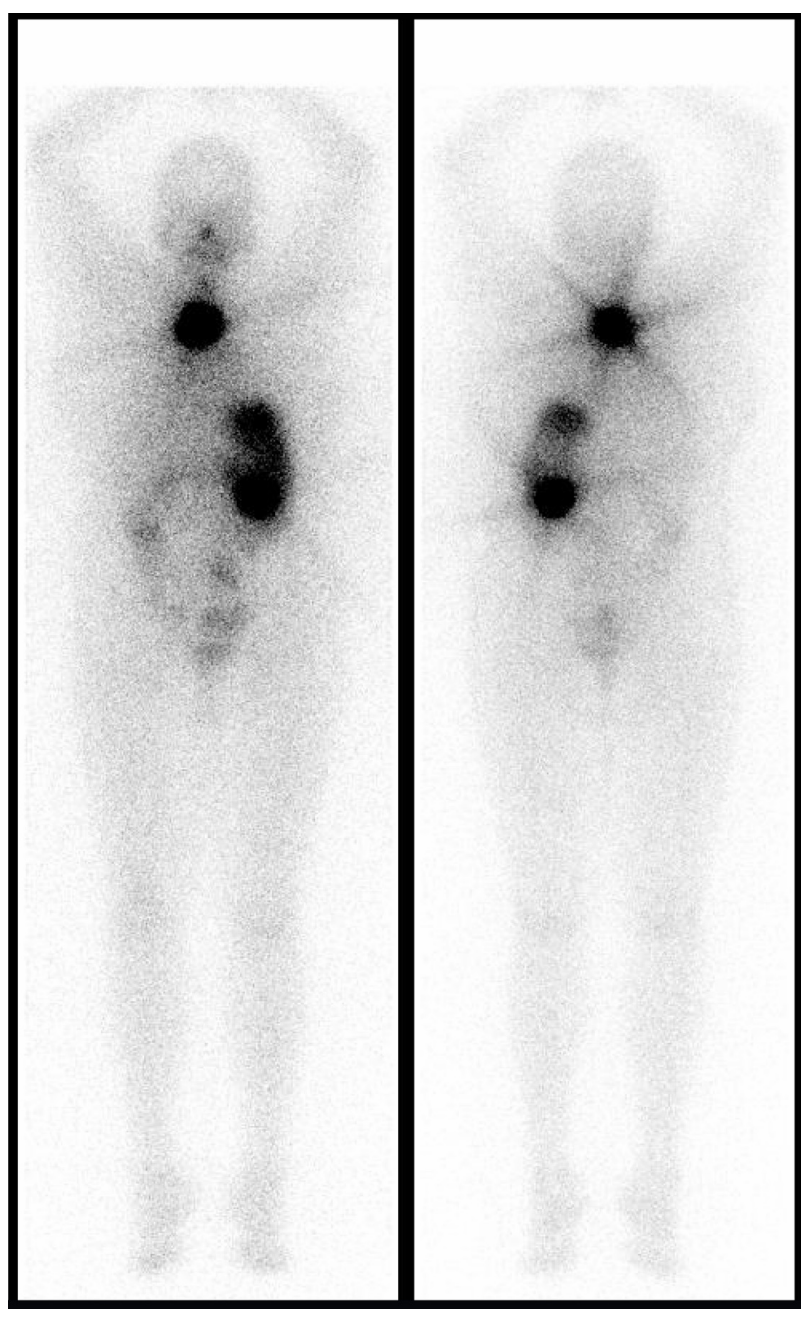

Figure 2 Preoperative ${ }^{|3|} \mid$-WBS.

Note: The exam showed uptaking vertebral and renal metastases.

Abbreviations: ${ }^{|3|} \mid$, iodine-|3|; WBS, whole body scan.

cancer with negative surgical margins (Figure 4). Furthermore, immunohistochemical analysis showed positive results for galectin-3, HBME1, and CK19.

After renal enucleation and stimulation with recombinant TSH, thyroglobulin was $82 \mathrm{ng} / \mathrm{mL}$, TSH was $15 \mathrm{mU} / \mathrm{L}$, and antithyroglobulin antibody was negative.

After 2 months, the patient underwent adjuvant radiometabolic treatment with ${ }^{131} \mathrm{I}(150 \mathrm{mCi})$ and the following WBS showed only a small uptaking area at the level of the vertebral metastasis (Figure 5). This finding proved a partial response of the bone lesions to radioiodine therapy. At 30 months, an abdominal CT scan did not detect any renal recurrence of tumor. At 36 months follow-up, the lung metastases were not detectable and the malignant disease was clinically stable and well-controlled. The clinical stage was T1bN0M1 stage IVC according to the 2009 TNM classification. ${ }^{7}$

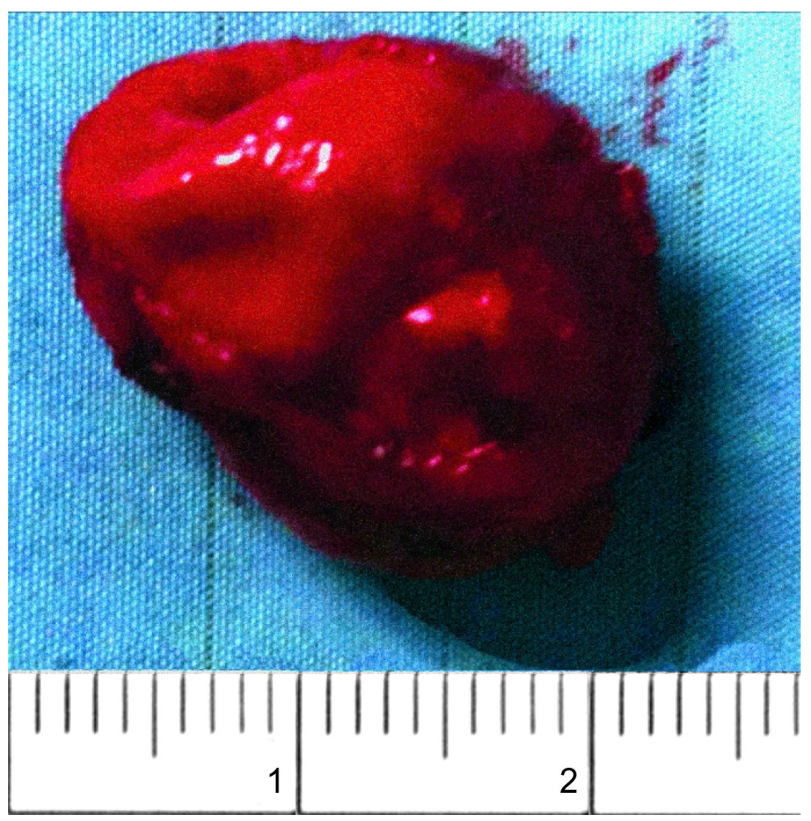

Figure 3 Surgical specimen of renal metastasis.

Notes: It is important to note that the tumor was completely removed and macroscopically the resection margins were negative. Moreover, the renal metastasis from thyroid cancer was macroscopically similar to primitive renal cancer.

\section{Discussion}

$10 \%-15 \%$ of patients with follicular carcinoma present with metastatic disease, most commonly involving the lung followed by bone. Brain, liver, and skin are less common sites of systemic metastases. ${ }^{5}$ Renal involvement from thyroid primary cancer is

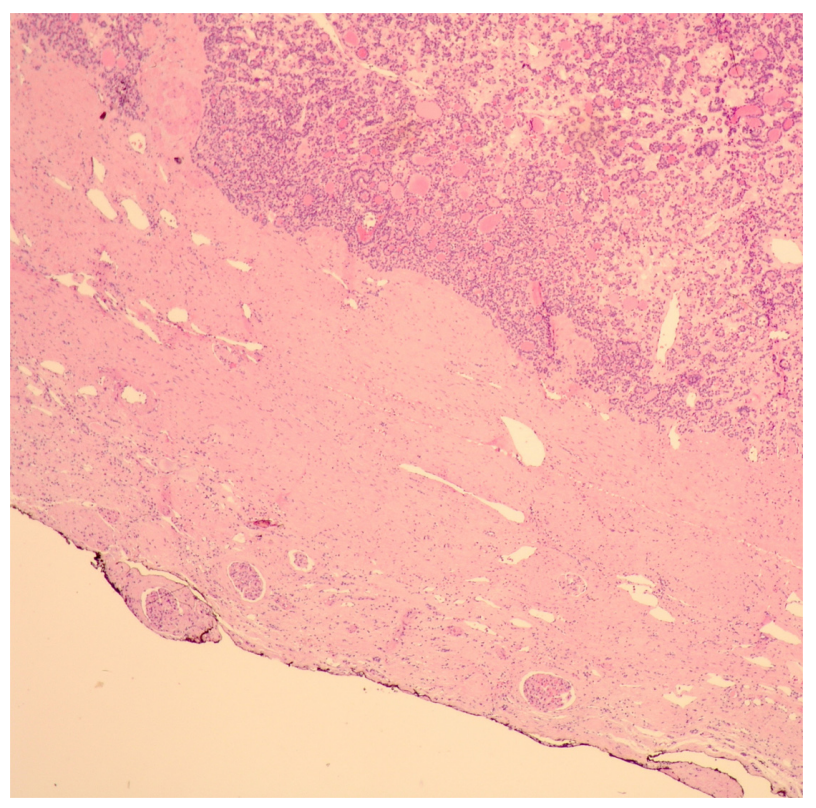

Figure 4 Histological findings.

Notes: Histological findings revealed the tumor cells were arranged in a well-differentiated follicular pattern. Colloid-like materials were found in the intrafollicular space. The resection margins were negative (hematoxylin and eosin staining: original magnification $\times 40$ ). 


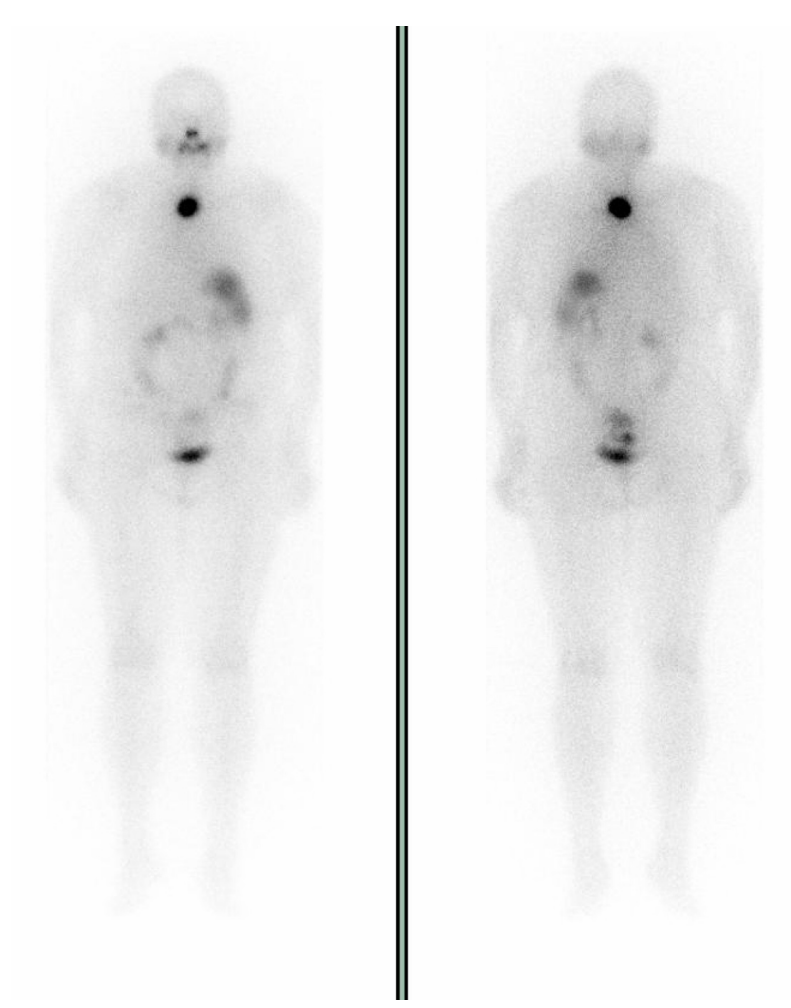

Figure 5 Post-partial nephrectomy ${ }^{|3|}$ I-WBS.

Notes: Small uptake of vertebral lesion is visible. Renal uptake was not detectable. Abbreviations: ${ }^{131}$ I, iodine-|3|; WBS, whole body scan.

very rare $(4.5 \%-5.9 \%)$. Only 18 cases of renal metastasis from thyroid carcinoma have been reported in the English literature and over 30 cases in the Japanese literature. ${ }^{8-12}$

Thyroid carcinoma accounts for only $2.5 \%-2.7 \%$ of all primary tumors that metastasize to the kidney. ${ }^{4}$ However, renal metastases are found more often at autopsy than during life. It could be related to a variable expression of sodium iodide symporter (NIS) among different metastatic sites or to the selective loss of function of the NIS. ${ }^{13}$ In our case, the metastatic focus was single and unilateral, contrary to what commonly occurs: the thyroid cancer metastasizes predominantly by a hematogenous path, resulting in multiple and bilateral renal metastases. This apparent anomaly may be related to the presence of venous and/or lymphatic minor collaterals between the thyroid gland and kidney. ${ }^{4}$ These metastases are often asymptomatic and their diagnosis may be delayed, especially if radiometabolic treatment is not performed or if the metastatic foci are not concentrating radioiodine. It is important to stress that a strong radiometabolic uptake in the upper abdominal tract should never be considered as a false positive or as physiological activity of the gastrointestinal tract, since biliary, gastrointestinal, and urinary radioiodine activity is much more diffuse. Therefore, any radioiodine activity detected by the radioiodine WBS should always be investigated further by anterior and posterior scans. Lateral views may also be obtained, as they could be helpful in distinguishing gastric or hepatic uptake from renal/adrenal uptake, which should be more posteriorly located. In our case, the strong uptake of radioiodine in the kidney not only allowed for the diagnosis of metastatic renal involvement, but also it significantly reduced the effectiveness of radiometabolic treatment of the secondary vertebral and lung injuries (Figure 1).

Management of renal metastasis from thyroid cancer includes radical nephrectomy combined with repeated highdose radioiodine therapies, possibly guided by whole body and blood dosimetry. ${ }^{14-17}$

Niederle et al reported on a total of 45 patients affected by cancer ( 41 follicular and 4 papillary carcinomas) that received surgical treatment for distant metastases. ${ }^{14}$ Fiftyfour metastatic lesions were removed, but no renal metastasis was reported. A total of 25 metastases (17 bone, eight soft tissue) were removed by resection. They reported that the estimated cumulative survival rate (Kaplan-Meier) was $44.8 \% \pm 11.2 \%$ for 5 years and $32.7 \% \pm 11.0 \%$ for 10 years after removal of a solitary metastasis. The study highlighted that the surgical removal of resectable metastases can be a valuable complement to nuclear medical therapy. ${ }^{14}$

Wood et al found that the 5-year survival period of welldifferentiated thyroid carcinoma patients with resectable metastatic lesions was $67 \%{ }^{18}$

$\mathrm{Xu}$ et al reported a case of a 64-year-old woman with metastatic thyroid follicular carcinoma presenting as a primary renal tumor; they performed radical nephrectomy and, after 6 months, a total thyroidectomy with a lymphadenectomy. ${ }^{8}$ They recommend that the treatment for well-differentiated follicular carcinoma with distant metastasis is maximal excision of the tumors followed by thyroid hormone supplementation and ${ }^{131}$ I therapy, because complete surgical excision of the distant metastatic lesion and primary tumor can still offer a good prognosis. ${ }^{8}$

Kumar et al treated renal metastasis by performing only high-dose radioiodine therapy as the patient refused surgery. ${ }^{4}$ When surgical excision is not possible, high-dose radioiodine therapy followed by a suppressive dose of L-thyroxine is a favorable approach because it prolongs survival by delaying disease progression. ${ }^{4}$

In our opinion, surgical exeresis of renal metastasis was necessary in this case in order to improve the effectiveness of radiometabolic treatment on bone and lung metastases. Indeed, surgery allowed a debulking of the surgical removable metastases, reducing the tumor burden to be treated by radioactive iodine. Moreover, it is estimated that ${ }^{131} \mathrm{I}$ therapy is 
effective in only $55 \%$ of patients with bone metastases and only $17 \%$ of them develops remission. ${ }^{19,20}$ Radiometabolic treatment can be curative in $35 \%-45 \%$ of micronodular pulmonary metastases with a reported 10-year survival rate of $90 \%{ }^{21}$

Therefore, in our case, the mini-invasive surgical management of the renal metastasis increased the chance that radioiodine therapy would be effective on the vertebral and lung metastases. Moreover, surgery has an important role in the management of metastatic disease: macroscopic metastases that are isolated to lung, bone, or brain that can be resected result in improved survival. ${ }^{5}$ In our case, after the combined approach with surgical removal of renal metastasis and radiometabolic treatment, the patient developed a remission of lung metastases, that were not detectable, and a stabilization of bone metastases. The WBS following adjuvant radioiodine therapy pointed out partial response of bone lesions to radiometabolic treatment with only a small uptaking area at the level of the vertebral lesions.

Despite a previous extended left hemicolectomy for colon rectal cancer, we performed a retroperitoneal laparoscopy to minimize intraoperative bleeding, to not stop bowel peristalsis, and to reduce postoperative pain. Consequently, this surgical strategy decreased the discomfort of the patient and the length of stay. We did not clamp the renal hilum to avoid damage related to ischemia of healthy renal parenchyma. As already demonstrated in the literature, the absence of intraoperative ischemia allows for better preservation of renal function in the long term. ${ }^{22}$

Moreover, longer warm ischemia time during partial nephrectomy is associated with an increased risk of short and long-term renal injuries, including acute renal failure in the postoperative period and new-onset development of stage IV chronic kidney disease during follow-up. ${ }^{23}$ Preservation of renal function could be useful to the following therapies. The mini-invasive surgery allowed mobilization of the patient at the first postoperative day, earlier recovery of general health, and earlier reinstatement of high-dose radioiodine treatment of the other metastases. To our knowledge, there are no studies about laparoscopic enucleation of renal metastasis from thyroid cancer. The complicated surgical treatment of metastasis is justified by the favorable effect on prognosis and the patient's quality of life.

\section{Conclusion}

To our knowledge, this is the first case of laparoscopic extraperitoneal simple enucleation of renal metastasis from thyroid cancer. This therapeutic management preserves renal function and improves the effectiveness of the following radioiodine treatment. When thyroid carcinoma metastasizes to the kidney, minimally invasive renal enucleation associated with repeated high-dose radioiodine treatment could be a new and useful approach to delay malignant disease progression, reducing the surgical discomfort of the patient. However, further studies with larger series are needed.

\section{Disclosure}

The authors report no conflicts of interest in this work.

\section{References}

1. Siegel R, Naishadham D, Jemal A. Cancer statistics, 2012. CA Cancer J Clin. 2012;62(1):10-29.

2. Wartofsky L. Increasing world incidence of thyroid cancer: increased detection or higher radiation exposure? Hormones (Athens). 2010; 9(2): 103-108.

3. Maxon HR 3rd, Smith HS. Radioiodine-131 in the diagnosis and treatment of metastatic well differentiated thyroid cancer. Endocrinol Metab Clin North Am. 1990;19(3):685-718.

4. Kumar A, Nadig M, Patra V, Srivastava DN, Verma K, Bal CS. Adrenal and renal metastases from follicular thyroid cancer. Br J Radiol. 2005; 78(935):1038-1041.

5. McHenry CR, Phitayakorn R. Follicular adenoma and carcinoma of the thyroid gland. Oncologist. 2011;16(5):585-593.

6. Shaha AR. Implications of prognostic factors and risk groups in the management of differentiated thyroid cancer. Laryngoscope. 2004; 114(3):393-402.

7. Sobin LH, Compton CC. TNM seventh edition: what's new, what's changed: communication from the International Union Against Cancer and the American Joint Committee on Cancer. Cancer. 2010; 116(22):5336-5339.

8. Xu H, Zeng W, Tang Y. Metastatic thyroid follicular carcinoma presenting as a primary renal tumor. Intern Med. 2012;51(16):2193-2196.

9. Iwai H, Ohno Y, Ito H, Kiyokawa T, Aoki N. Renal rupture associated with a poorly differentiated follicular thyroid carcinoma metastasizing to the thigh muscle, lung and kidney. Intern Med. 2005;44:848-852.

10. Moudouni SM, En-Nia I, Rioux-Leclerq N, et al. Follicular carcinoma of the thyroid metastasis to the kidney nine years after resection of the primary tumor. Ann Urol. 2002;36:36-37.

11. Liou MJ, Lin JD, Chung MH, et al. Renal metastasis from papillary thyroid microcarcinoma. Acta Otolaryngol. 2005;125:438-444.

12. Djekidel M, Gordon M, Shah RB, et al. Renal metastasis from Hurthle cell thyroid carcinoma and its evaluation with hybrid imaging. Thyroid. 2010;20:429-433.

13. Smallridge RC, Castro MR, Morris JC, et al. Renal metastases from papillary thyroid carcinoma: study of sodium iodide symporter expression. Thyroid. 2001;11(8):795-804.

14. Niederle B, Roka R, Schemper M, Fritsch A, Weissel M, Ramach W. Surgical treatment of distant metastases in differentiated thyroid cancer: indication and results. Surgery. 1986;100(6):1088-1097.

15. Pak H, Skarulis MC, Schrump D, Alexander HR, Libutti SK, Sarlis NJ. The role of extensive metastatectomy in the long term management of clinically aggressive thyroid carcinoma. The NIH experience. Proceedings of the 11th International Congress of Endocrinology; 2000; Sydney, Australia; P803 [Abstract].

16. Reynolds JC, Robbins J. The changing role of radioiodine in the management of differentiated thyroid cancer. Semin Nucl Med. 1997; 27(2):152-164.

17. Maxon HR. Quantitative radioiodine therapy in the treatment of differentiated thyroid cancer. Q J Nucl Med. 1999;43(4):313-323.

18. Wood WJ, Singletary SE, Hickey RC. Current result of treatment for distant metastatic well-differentiated thyroid carcinoma. Arch Surg. 1989;124:1374-1377. 
19. Durante C, Haddy N, Baudin E, et al. Long-term outcome of 444 patients with distant metastases from papillary and follicular thyroid carcinoma: benefits and limits of radioiodine therapy. J Clin Endocrinol Metab. 2006;91(8):2892-2899.

20. Proye CA, Dromer DH, Carnaille BM, et al. Is it still worthwhile to treat bone metastases from differentiated thyroid carcinoma with radioactive iodine? World J Surg. 1992;16(4):640-645.

21. Schlumberger M, Tubiana M, De Vathaire F et al. Long-term results of treatment of 283 patients with lung and bone metastases from differentiated thyroid carcinoma. J Clin Endocrinol Metab. 1986;63:960 -967.
22. Aron M, Gill IS, Campbell SC. A nonischemic approach to partial nephrectomy is optimal. Yes. J Urol. 2012;187(2):387-388.

23. Thompson RH, Lane BR, Lohse CM, et al. Every minute counts when the renal hilum is clamped during partial nephrectomy. Eur Urol. 2010; 58(3):340-345.

\section{Publish your work in this journal}

OncoTargets and Therapy is an international, peer-reviewed, open access journal focusing on the pathological basis of all cancers, potential targets for therapy and treatment protocols employed to improve the management of cancer patients. The journal also focuses on the impact of management programs and new therapeutic agents and protocols on

\section{Dovepress}

patient perspectives such as quality of life, adherence and satisfaction. The manuscript management system is completely online and includes a very quick and fair peer-review system, which is all easy to use. Visit http://www.dovepress.com/testimonials.php to read real quotes from published authors.

Submit your manuscript here: http://www.dovepress.com/oncotargets-and-therapy-journal 